\title{
Pilot Study on an Integrated Pilates and Yoga Program for Decreasing Postpartum Depression in Women
}

\author{
Yi-Li Ko1* ${ }^{*}$ Pi-Chu Lin², Chi-Li Yang ${ }^{3}$, Chie-Pein Chen", Huai-Jung Shih ${ }^{5}$ \\ ${ }^{1}$ Department of Nursing, College of Medicine, Fu-Jen Catholic University, Taiwan \\ ${ }^{2}$ School of Nursing and Master Program in Long-Term Care, College of Nursing, Taipei Medical University, \\ Taiwan \\ ${ }^{3}$ Department of Physical Education, Shih Chien University, Taiwan \\ ${ }^{4}$ Department of Obstetrics and Gynecology, Mackay Memorial Hospital, Taiwan \\ ${ }^{5}$ Department of Dentistry, University of Szeged, Szeged, Hungary \\ Email: \\ sunplay77712@hotmail.com
}

Received 24 September 2015; accepted 23 October 2015; published 26 October 2015

Copyright (C) 2015 by authors and Scientific Research Publishing Inc.

This work is licensed under the Creative Commons Attribution International License (CC BY).

http://creativecommons.org/licenses/by/4.0/

c) (i) Open Access

\begin{abstract}
Objective: This study evaluated the effectiveness of a 10 -week exercise intervention in reducing depression and fatigue in women with postpartum depression. Design: A one-group pretest/ posttest quasi-experiment was conducted. Setting: A postpartum ward in a medical center in Taipei city was used. Participants: Nineteen women at 7 - 12 weeks postpartum with an Edinburgh Postnatal Depression Scale score $\geq 9$ were recruited. Methods: The women participated in a postpartum exercise support program 1 hour per week for 10 weeks. The program was led by a professional coach; it integrated yoga, Pilates, elastic band exercise, low-intensity aerobics, and motherhood role experience sharing. Results: After the postpartum exercise support program intervention, depression scores decreased from $10.4 \pm 3.29$ to $7.80 \pm 2.73(t=3.632, P=0.003)$; fatigue scores decreased from $8.73 \pm 5.02$ to $5.40 \pm 3.89(t=2.988, P=0.010)$. Conclusion: PESP administered to women with postpartum depression appeared to benefit their psychological wellbeing.
\end{abstract}

\section{Keywords}

Exercise, Women with Postpartum Depression, Fatigue, Depression

\footnotetext{
"Corresponding author.
}

How to cite this paper: Ko, Y.-L., Lin, P.-C., Yang, C.-L., Chen, C.-P. and Shih, H.-J. (2015) Pilot Study on an Integrated Pilates and Yoga Program for Decreasing Postpartum Depression in Women. Open Journal of Nursing, 5, 885-892. 


\section{Introduction}

Postpartum depression (PPD) is a prominent health issue. Few multidisciplinary studies, combining physicalexercise and nursing, have been conducted. The prevalence of PPD reports $10 \%-15 \%$ in Western country [1]. In Taiwan, a 40\% prevalence of mild-to-severe PPD has been reported at 6 weeks postpartum [2]. In a metaanalysis of 30 studies based only on structured clinical interview assessments, the point prevalence is estimated to be approximately $6.5 \%-12.9 \%$, involving both major and minor depression at various times during the first postpartum year [3]. PPD is characterized by episodes of irritability, insecurity, anxiety, fatigue, guilt, obsessive thoughts, and physical symptoms, which have negative effects on mother-infant interactions [4] [5]. Research has determined that $44 \%$ - 95\% of postpartum mothers in the United States experience postpartum fatigue [6] [7]. Postpartum fatigue is also a common problem in Taiwan [8] [9]. Postpartum fatigue is a predictor of PPD [9]. Postpartum psychological problems can interfere with a new mother's ability to care for her child and may adversely affect her quality of life [9].

In Taiwan, if postpartum women exhibit depressive symptoms, physicians often consult psychiatrists to prescribe antidepressants; however, mothers are typically worried about exposing infants to the adverse side effects of drugs through breastfeeding; they are generally afraid to take medicine. Epidemiological research has suggested that physical activity may be associated with reduced symptoms of depression. Few studies have investigated the effect of exercise programs on women's psychological health during the postpartum period. However, concerns have been expressed regarding the methodological quality of these studies [10]-[12]. In Taiwan, only one study has been conducted on PPD intervention [12]. Heh evaluated whether an exercise program (consisting of one hour-long session per week at a hospital and two sessions per week at home for 3 months) was more effective than routine care for alleviating depression symptoms in women with an Edinburgh Postnatal Depression Scale (EPDS) score $>10$ [12]. The results indicated that women with PPD $(n=33)$ who attended an exercise support program were less likely to exhibit high depression scores at 5 months postpartum than the women in the control group $(n=30)$. Da Costa et al. examined 88 Women with PPD who underwent a 12-week home-based exercise program and determined that the program constituted an effective intervention for alleviating postpartum depressive symptoms, particularly in women with greater depression at baseline (EPDS; score $>13$ ). The intervention group exhibited a significantly lower post-treatment EPDS score than the usual-care group did (mean difference 4.06 points, 95\% confidence interval $1.51-6.61, p<0.001$ ) [11]. Armstrong and Edwards developed a 12-week pram-walking exercise program (for mothers and their babies) and compared the outcomes to those of a social support group (nonstructured session, similar to a playgroup). The results suggested that members of the pram-walking group reduced their level of depressive symptomatology significantly more than the members of the social support group did [10].

Chinese culture considers "postpartum preservation" as critical to the overall wellness of a woman who has given birth. In Chinese culture, postpartum women should eat high-calorie and high-protein food, rest, and avoid the outdoor environment in addition to caring for their infants. In this period, mothers may reduce or stop exercising, which can affect subsequent exercise habits [13]. Ko et al. [14] developed a community-based "Yoga and Pilates Exercise Program” for general postpartum women (i.e. not targeted at specifically women with PPD) in Taiwan. The exercise program was conducted in group sessions of 60 min, once a week for 3 months (12 times in total) by a professional coach. A total of 23 women at 2 - 6 months postpartum completed the entire program and it was determined that the program was effective in improving the women's PPD. This study used only a small sample size, and the authors found that it was difficult to encourage women to go outdoors for exercise because of the traditional ideas about postnatal care in Taiwan.

Despite limited evidence regarding women with PPD, the National Institute for Health and Clinical Excellence in the United Kingdom recommended in their postnatal mental-health guidelines that health professionals should consider exercise as a management strategy for women experiencing mild-to-moderate PPD.

The purpose of this study was to evaluate the effectiveness of a postpartum exercise support program (PESP) in reducing fatigue and depression in women with PPD.

\section{Materials and Methods}

\subsection{Study Design}

This study adopted a quasi-experimental one-group pretest/posttest design and a convenience sampling method. 
Study participants, who were at 7 - 12 weeks postpartum, participated in a 10-week exercise program developed in this study, one session perweek.

\subsection{Participants and Setting}

Participants were recruited between May and July 2013 at a medical center in Taipei. The inclusion criteria were as follows: being postpartum, aged between 20 and 40 years, exhibiting no maternal or neonatal complications, having an EPDS score $\geq 9$, and willing to participate in the study.

Research assistants (RAs) used the EPDS to assess women at 1 - 3 days after giving birth in the postpartum ward. When women exhibited an EPDS score $\geq 9$, Ras obtained written consent after explaining research the purpose and process, and left a contact telephone number. Researchers arranged women to participate in a PESP until 7 - 12 weeks postpartum.

After the initial recruited, 50 women with an EPDS score $\geq 9$ accepted the invitation; however, after 1 - 2 months, having completed 1 month, several women left the program, causing a small sample size in this study. The reasons of withdrawal from the study included the following: women were living in Southern Taiwan (their parents' home, which was far from the study site); women had to work on the day of class; women felt tired, had no motivation, and did not feel comfortable letting others take care of their baby.

Overall, 19 women were enrolled, 15 of whom completed the entire program and answered the questionnaires (a $79 \%$ valid response rate). Four women did not complete the 10-week exercise program for work or childcare reasons.

\subsection{Experimental Intervention}

\subsubsection{Postpartum Exercise Support Program}

The PESP was designed for women who were considered to have mild PPD. The exercise support program consisted of the following: 1) 10-minute warm-up and 10-minute cool-down; 2) a 40-minute whole body stretching exercise, integrated Pilates, yoga, and elastic band exercise, which achieved a target heart rate (HR) of $60 \%-75 \%$ of maximal HR; 3) after-exercise program with a 30-minute experience-sharing session, including breastfeeding and talking about adapting to the new role as a mother; 4) RAs created an exercise program video and upload edit to Facebook; the participants were encouraged to practice the exercises based on the video at home at least twice perweek. In addition, the RAs reminded the participants to attend the exercise class every week using Line (a mobile device application).

\subsubsection{Professional Coach-Led Program}

The postpartum exercise program was led by a professional coach who was one of researchers. The coach has a doctor of philosophy degree and is licensed to teach postpartum yoga and Pilates. The postpartum exercise program was conducted in a group once per week; it lasted from 10:30 to 11:30 a.m. every Saturday for a 3-month period (total 10 sessions) in a gym at a university.

\subsubsection{Facilitators}

Ras used Line to maintain weekly contact with the postpartum women to monitor home exercise, provide remote care, and remind the women to continue with the program. To encourage participation: a gift was presented after each week (e.g. a pedometeror gift certificate).

The stadium is a professional venue with wooden floor classrooms and spacious, comfortable areas; there is a beautiful surrounding landscape with numerous trees and large areas of grassland that provide bright and sunny spaces. The researcher and Ras joined the weekly program, to help with taking care of the babies when necessary; in addition, female family members of the participants were allowed to attend classes.

\subsection{Instruments}

The study tools comprised a structured questionnaire designed in this study, including basic information, postpartum exercise status, the Fatigue Symptoms Checklist (FSC), and the EPDS.

Basic Information: The basic information obtained was age, height, prepregnancy weight, weight gain during pregnancy, current weight, education level, occupation, family type, annual household income, pregnancy his- 
tory, childbirth history, and the current infant feeding method.

Postpartum Exercise Status: The data obtained were used to evaluate whether participants were engaging in regular exercise, their exercise intensity, exercise conditions, and barriers to exercise after giving birth.

FSC: The FSC, developed by Yoshitake, [15] was designed for the National Institute of Occupational Safety and Health in Japan to assess fatigue symptoms. The checklist contains 30 common fatigue symptoms that are categorized into three categories as follows: sleepy and powerless (Items 1 - 10), difficulty concentrating (Items 11 - 20), and partial abnormalities of the body (Items 21 - 30). Each item permits a dichotomous yes/no (0 to 1) response. Possible scores range between 0 and 30, and a high score is indicative of severe fatigue.

The FSC is a multidimensional scale that exhibits strong reliability and validity and is not excessively taxing to complete. Milligan et al. [7] used the FSC to measure postpartum fatigue among 285 mothers immediately after birth, during the hospitalization period, and 6 weeks postpartum. Their results indicated that the internal consistency of the scale ranged from 0.82 to 0.95 .

EPDS: The EPDS has been determined to exhibit higher validity than the BDI regarding sensitivity and specificity [16] [17]. The EPDS consists of 10 short statements and requires less than 5 minutes to complete. A cutoff score of 9 is recommended for identifying genuine cases of postnatal depression [16].

Study Tools and Tests of Reliability and Validity: The scales adopted in this study were widely used domestic and international scales, all of which exhibit good reliability. In this study, the Cronbach $\alpha$ obtained for both the FSC and the EPDS was 0.86 .

\subsection{Ethical Considerations}

This study was approved by the institutional review board of the hospital where the study was conducted (IRB No. 12MMHIS161). The purpose and process were explained to the participants, and after signing an informed consent form, they were enrolled in the study. In addition, participants were informed that they could withdraw from the study at any time without a loss of benefits. The women were assured that involvement in the study would not affect their medical care and that all information obtained from the questionnaire would be treated confidentially and used only for research purposes.

\subsection{Data Collection Procedure}

Our RA recruited eligible women with PPD in the postpartum ward in a medical center in Taipei by describing the purpose of the study. Because Taiwanese have a "doing-the-month" culture whereby, after delivery, postpartum women are discharged from the hospital and expected to stay at home for an entire month; therefore, our RA called the women regarding follow-up care during this month.

A pretest was conducted before the first session of the exercise program. The pretest was used to collect basic participant information, postpartum exercise habits, postpartum fatigue, and body composition. After the participants had attended 10 sessions of the program, a posttest was conducted to obtain measurements regarding postpartum fatigue and depression.

\subsection{Data Analysis}

SPSS version 19.0 was used to analyze data and significant level was set as 0.05 (SPSS, Chicago, IL, USA). Participants' basic information was exhibited according to frequency, percentage, mean, and standard deviation (SD). A paired $t$ test was employed to identify differences in fatigue and depression before and after the exercise program.

\section{Results}

\subsection{Basic Participant Information}

The average age of the 15 postpartum women was $34.3 \pm 3.8$ years. The average number of pregnancies among the participants was $1.27 \pm 0.46$; 12 participants (80\%) were primiparas. All participants had a college degree or above (100\%). In addition, most participants had a full-time job (60\%). Twelve (80\%) participants had NSDs, whereas 20\% had CS. Six (40\%) participants breastfed exclusively, and the others adopted mixed feeding methods. Only four (26.7\%) of the participants exercised after childbirth. Six of the participants (40\%) lived in a 
nuclear family (Table 1).

\subsection{Depression and Fatigue of Participants}

The mean score of depression in the pretest was $10.40 \pm 3.29$ points; the scores ranged between 0 and 30 . Three women (20\%) scored $\geq 12$ points on the EPDS. The mean depression score was $7.80 \pm 2.73$ points in the posttest. The decrease of 2.60 points was significant $(t=3.632, P=0.003)$.

The mean score of fatigue in the pretest was $8.73 \pm 5.02$ points, and $5.40 \pm 3.89$ points in the posttest. A paired $t$ test was used to compare feelings of fatigue before and after the exercise intervention; the decrease of 3.33 points was significant $(t=2.988, P=0.010)$. Comparing the scores of fatigue subscales revealed significant differences in physical and psychological scores before and after the intervention $(t=2.525, P=0.024 ; t=2.824$, $P=0.014)$. No significant difference was observed in the symptom score before and after the test $(t=1.234, P=$ 0.238 ; Table 2).

Table 1. Basic information of study participants $(n=15)$.

\begin{tabular}{|c|c|c|}
\hline Characteristic & $n(\%)$ & $M \pm S D$ \\
\hline Age (year) & & $34.33 \pm 3.84$ \\
\hline Gravida & & $1.27 \pm 0.46$ \\
\hline Primipara & $12(80 \%)$ & \\
\hline \multicolumn{3}{|l|}{ Education level } \\
\hline$\geqslant$ University & $13(86.7 \%)$ & \\
\hline$\leqslant$ College & 2 (13.3\%) & \\
\hline \multicolumn{3}{|l|}{ Work status } \\
\hline —Full-time job & $9(60 \%)$ & \\
\hline —Housewife & $6(40 \%)$ & \\
\hline \multicolumn{3}{|l|}{ Method of delivery } \\
\hline$-\mathrm{NSD}$ & $12(80 \%)$ & \\
\hline —Cesarean section & $3(20 \%)$ & \\
\hline \multicolumn{3}{|l|}{ Breastfeeding } \\
\hline —Exclusive & $6(40 \%)$ & \\
\hline —Formula & $1(6.7 \%)$ & \\
\hline —Mixed feeding & $8(53.3 \%)$ & \\
\hline \multicolumn{3}{|l|}{ Exercise habits (pretest) } \\
\hline —Yes & $4(26.7 \%)$ & \\
\hline$-\mathrm{No}$ & $11(73.3 \%)$ & \\
\hline \multicolumn{3}{|l|}{ Family structure } \\
\hline —Nuclear family & $6(40 \%)$ & \\
\hline -Other & $9(60 \%)$ & \\
\hline
\end{tabular}

Note. NSD: normal spontaneous delivery; C: section, cesarean section.

Table 2. Participants depression and fatigue scores $(n=15)$.

\begin{tabular}{ccccc}
\hline Dimensions and subscales & $\begin{array}{c}\text { Pretest } \\
M \pm S D\end{array}$ & $\begin{array}{c}\text { Post-test } \\
M \pm S D\end{array}$ & $t$ & $P$ \\
\hline Fatigue & & & & \\
- Physical (items 1 - 10) & $4.13 \pm 2.13$ & $2.40 \pm 1.77$ & 2.525 & $0.024^{*}$ \\
- Psychological (items 11 - 20) & $2.40 \pm 2.20$ & $1.47 \pm 1.77$ & 2.824 & $0.014^{*}$ \\
- Symptoms (items 21 - 30) & $2.20 \pm 2.15$ & $1.53 \pm 1.51$ & 1.234 & 0.238 \\
- Total score & $8.73 \pm 5.02$ & $5.40 \pm 3.89$ & 2.988 & $0.010^{*}$ \\
Depression scores $(n=15)$ & $10.40 \pm 3.29$ & $7.80 \pm 2.73$ & 3.632 & $0.003^{*}$ \\
\hline
\end{tabular}

${ }^{*} \mathrm{P}<0.05 ;{ }^{* *} \mathrm{P}<0.001$. 


\section{Discussion}

In this study, $20 \%$ of participants who were 7 - 12 weeks postpartum exhibited a depression score of $\geq 12$ points in the pretest, which is the cutoff point indicating depression in Taiwan; this suggests that these women were prone to depression. However, the depression scores obtained in this study were lower than those obtained by Chen's et al. study, [2] who found that in Taiwan, 40\% of women 6 weeks postpartum received a diagnosis of postnatal depression. Robertson et al. reported that the prevalence rate of PPD was 30\% - 75\% [18], which is higher than that exhibited in this study. The occurrence of depressive illnesses following childbirth can be detrimental to mothers, marital relationships, and children, and can have adverse long-term effects if left untreated. Certain studies have indicated that postpartum moods are associated with changes in hormone levels, including progesterone, estrogen, cortisone, beta-endorphins, oxytocin, and prolactin [17]; therefore, many postpartum women experience depression. In addition, psychosocial factors play key roles; Robertson et al. indicated that the most critical period for detecting postnatal depression is within 6 weeks of delivery [18]. Daley, MacArthur, and Winter stated that the peak incidence of depression is within the first 4 - 6 weeks after giving birth [19]. When hormone levels stabilize, depressive moods may improve. The period of data collection in this study was 7 - 12 weeks after delivery; therefore, the prevalence of depression should have been lower than that demonstrated immediately after delivery.

The results of this study demonstrated that our PESP reduced physical and psychological fatigue and depression. This finding is consistent with those reported by previous studies, they suggested that exercise reduces depression severity [12] [20]. Heh et al. investigated the effectiveness of exercise in reducing the severity of postnatal depression. In their study, 80 primiparas in Taiwan exhibiting an EPDS score of $>10$ at 4 weeks postpartum were allocated into either an intervention group (provided with exercise support) or a control group (provided with standard care) at 6 weeks postpartum. The main measured outcome was the EPDS score at 5 months postpartum. The results revealed that women who participated in the exercise support program were less likely to exhibit high depression scores after childbirth than the women in the control group $(P=0.01)$. By contrast, among participants in the low-score group, the exercise program did not result in any significant differences in depression scores [12]. Daley et al. reviewed five RCT or quasi-RCT studies that targeted postnatal depression management intervention for treating postpartum women. The results showed that compared with participants who did not exercise, in participants who did exercise, symptoms of postnatal depression were reduced (obtaining a standardized mean difference in effect of $-0.8 \%$ and a $95 \%$ confidence interval from -1.53 to -0.10 ) [20].

Dritsa, et al. indicated that exercise reduced tension and raised exercise-related energy consumption, thereby relieving postpartum fatigue [21]. Researchers have suggested that a proportion of postpartum fatigue depends on the characteristics of the infant and breastfeeding behavior, [22] and exercise may be ineffective regarding those factors. However, this study's finding was consistent with that of Dritsa et al.'s study [21]. The participants exhibited a significant relieve off atigue after 3 months of participating in the exercise program.

In addition, in the after-program experience-sharing sessions, postpartum women stated that participating in the exercise program had the following advantages: the location was near an Mass Rapid Transit (MRT) station; the teacher and venue were professional; the program content was interesting; the surroundings were beautiful and helped participants to relax; the elastic band could be taken home and used to exercise there; there was a full-time assistant to help take care of babies during exercises; and after they completed the PESP program, the women received a gift voucher. The intimate design of this program can be adapted for future interventions to enhance the recruitment rate.

\section{Conclusions}

The results of this study indicate that among women who are 7 - 12 weeks postpartum, the 10-week exercise intervention significantly reduces the fatigue and depression levels in those who are prone to depression.

This is the first trial study of women with PPD at 7 - 12 weeks postpartum who participate in a PESP in a gym. The results of this study are limited by the single-group pretest/posttest design, a study length of only 10 weeks, and a small sample size; therefore, the results should be generalized with caution. Encouraging women with PPD to leave their homes to engage in exercise is difficult. Therefore, recruiting numerous women with PPD who are willing to attend an exercise program is difficult. Further research that adopts a quasi-experimental design and involves a control group to enable comparing the effectiveness is necessary. In addition, the sample size and amount of exercise can be increased to improve the generalizability of the results. Regarding the high 
loss of participants in this study, future studies should consider how to encourage women with depressive tendencies to leave their homes to participate in an exercise program.

\section{Acknowledgements}

I hereby, thank all the pregnant women who participate in this research for their cooperation. The financial support from the National Science Council (NSC 101-2410-H-030-085) is greatly appreciated.

\section{References}

[1] Sierra Manzano, J.M. (2002) Variables Associated with the Risk of Postpartum Depression. Edinburgh Postnatal Depression Scale. Atención Primaria, 30, 101-111.

[2] Chen, C.H., Tseng, Y.F., Wang, S.Y. and Lee, J.N. (1994) The Prevalence and Predictors of Postpartum Depression. The Journal of Nursing Research, 2, 263-274.

[3] Gaynes, B.N., Gavin, N., Meltzer-Brody, S., Lohr, K.N., Swinson, T., et al. (2005) Perinatal Depression: Prevalence, Screening Accuracy, and Screening Outcomes. Agency for Healthcare Research and Quality, Rockville.

[4] Logsdon, M.C., Wisner, K.L. and Pinto-Foltz, M.D. (2006) The Impact of Postpartum Depression on Mothering. Journal of Obstetric Gynecology and Neonatal Nursing, 35, 652-653. http://dx.doi.org/10.1111/j.1552-6909.2006.00087.x

[5] Manchon, C.A., Barnett, B., Kowalenko, N.M. and Tennant, C.C. (2006) Maternal Attachment State of Mind Moderates the Impact of Postnatal Depression on Infant Attachment. Journal of Child Psychology and Psychiatry, 47, 660669. http://dx.doi.org/10.1111/j.1469-7610.2005.01547.x

[6] Atkinson, L.S. and Baxley, E.G. (1994) Postpartum Fatigue. American Academy of Family Physicians, 50, $113-118$.

[7] Milligan, R.A., Rarks, P.L., Kitzman, H. and Lenz, E.R. (1997) Measuring Women's Fatigue during the Postpartum Period. Journal of Nursing Measurement, 5, 3-16.

[8] Chen, C.H., Wang, S.Y., Chung, U.L. and Seng, Y.F. (2006) Being Reborn: The Recovery Process of Postpartum Depression in Taiwanese Women. Journal of Advanced Nursing, 54, 450-456. http://dx.doi.org/10.1111/j.1365-2648.2006.03843.x

[9] Ko, Y.L. (2004) Postpartum Fatigue. The Journal of Nursing, 51, 75-79. (In Chinese)

[10] Armstrong, K. and Edwards, H. (2004) The Effectiveness of a Pram-Walking Exercise Programme in Reducing Depressive Symptomatology for Postnatal Women. International Journal of Nursing Practice, 10, 177-194. http://dx.doi.org/10.1111/j.1440-172X.2004.00478.x

[11] Da Costa, D., Lowensteyn, I., Abrahamowicz, M., Ionescu-Ittu, R., Dritsa, M., et al. (2009) A Randomized Clinical Trial of Exercise to Alleviate Postpartum Depressed Mood. Journal of Psychosomatic Obstetrics \& Gynecology, 30, 191-200. http://dx.doi.org/10.1080/01674820903212136

[12] Heh, S.S., Huang, L.H., Ho, S.M., Fu, Y.Y. and Wang, L.L. (2008) Effectiveness of Exercise Support Program in Reducing the Severity of Postnatal Depression in Taiwan Women. Birth, 35, 60-65. http://dx.doi.org/10.1111/j.1523-536X.2007.00192.x

[13] Ko, Y.L., Yang, C.L. and Chiang, L.C. (2008) Effects of Postpartum Exercise Program on Fatigue and Depression during Doing the Month Period. Journal of Nursing Research, 16, 177-186. http://dx.doi.org/10.1097/01.JNR.0000387304.88998.0b

[14] Ko, Y.L., Yang, C.L., Fang, C.L., Lee, M.Y. and Lin, P.C. (2013) Community-Based Postpartum Exercise Program. Journal of Clinical Nursing, 22, 2122-2131. http://dx.doi.org/10.1111/jocn.12117

[15] Yoshitake, H. (1971) Relations between the Symptoms and the Feeling of Fatigue. Ergonomics, 14, 175-186. http://dx.doi.org/10.1080/00140137108931236

[16] Cox, J.L., Holden, J.M. and Sagovsky, R. (1987) Detection of Postnatal Depression Development of the 10-Iten Edinburgh Postnatal Depression Scale. The British Journal of Psychiatry, 150, 782-786. http://dx.doi.org/10.1192/bjp.150.6.782

[17] Harris, B., Huckle, P., Thomas, R., Johns, S. and Fung, H. (1989) The Use of Rating Scales to Identify Post-Natal Depression. The British Journal of Psychiatry, 154, 813-817. http://dx.doi.org/10.1192/bjp.154.6.813

[18] Robertson, E., Grace, S., Wallington, T. and Stewart, D.E. (2004) Antenatal Risk Factors for Postpartum Depression: A Synthesis of Recent Literature. General Hospital Psychiatry, 26, 289-295. http://dx.doi.org/10.1016/j.genhosppsych.2004.02.006

[19] Daley, A.J., MacArthur, C. and Winter, H. (2007) The Role of Exercise in Treating Postpartum Depression: A Review of the Literature. Journal of Midwifery \& Women's Health, 52, 56-62. http://dx.doi.org/10.1016/j.jmwh.2006.08.017 
[20] Daley, A., Jolly, K. and MacArthur, C. (2009) The Effectiveness of Exercise in the Management of Post-Natal Depression: Systematic Review and Meta-Analysis. The Journal of Family Practice, 26, 154-162. http://dx.doi.org/10.1093/fampra/cmn101

[21] Dritsa, M., Dupuis, G., Lowensteyn, I. and Da Costa, D. (2009) Effects of Home-Based Exercise on Fatigue in Postpartum Depressed Women: Who Is More Likely to Benefit and Why? Journal of Psychosomatic Research, 67, 159163. http://dx.doi.org/10.1016/j.jpsychores.2009.01.010

[22] Ko, Y.L. and Lu, C.M. (2003) Psychophysiologic and Situational Correlates of Postpartum Fatigue. Journal of Health Education, 19, 137-153. 